\title{
MicroRNA-542-3p represses OTUB1 expression to inhibit migration and invasion of esophageal cancer cells
}

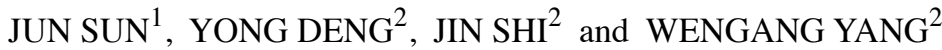 \\ ${ }^{1}$ Oncology Department, Jianhu Hospital Affiliated to Nantong University, Jianhu, Jiangsu 224700; \\ ${ }^{2}$ Thoracic Surgery Department, Sheyang People's Hospital, Sheyang, Jiangsu 224300, P.R. China
}

Received August 20, 2018; Accepted April 24, 2019

DOI: $10.3892 / \mathrm{mmr} .2019 .10836$

\begin{abstract}
Dysregulation of microRNAs (miRNAs) is involved in the pathogenesis of esophageal cancer. miRNA (miR)-542-3p is a tumor suppressor in multiple types of cancer. However, whether and how miR-542-3p contributes to the progression of esophageal cancer remains unknown, and this is the aim of the present study. In the current study, decreased expression of miR-542-3p was detected in tumor tissues compared with normal tissues from patients with esophageal cancer, and miR-542-3p expression was negatively correlated with mRNA expression levels of ovarian tumor domain-containing ubiquitin aldehyde-binding protein 1 (OTUB1) in tumor tissues from patients with esophageal cancer. In KYSE150 human esophageal squamous cell carcinoma cells, overexpression of miR-542-3p significantly decreased $O T U B 1$ at mRNA and protein levels, whereas downregulation of miR-542-3p significantly increased $O T U B 1$ expression. Using a dual-luciferase assay, $O T U B 1$ was validated to be a target gene of miR-542-3p in KYSE150 cells. Functionally, miR-542-3p significantly inhibited the migration and invasion of KYSE150 cells by repression of $O T U B 1$ expression. These results demonstrated that miR-542-3p may promote the metastasis of esophageal cancer cells, and indicated that miR-542-3p may be a treatment target for esophageal cancer.
\end{abstract}

\section{Introduction}

Esophageal cancer is the sixth most commonly diagnosed cancer and the fifth leading cause of cancer motility globally (1). It was estimated that esophageal cancer accounted for $>4 \%$ of cancer-related mortality in the USA in 2017 (2). In East Asia, the risk of incidence for esophageal cancer is almost 4-fold higher compared with North America (1). The two major types of esophageal cancer are esophageal squamous-cell

Correspondence to: Dr Wengang Yang, Thoracic Surgery Department, Sheyang People's Hospital, 27 Youhong Street, Sheyang, Jiangsu 224300, P.R. China

E-mail: yangwengangsyph@yandex.com

Key words: microRNA-542-3p, ovarian tumor domain-containing ubiquitin aldehyde-binding protein 1 , esophageal cancer carcinoma and esophageal adenocarcinoma (3). Owing to its aggressive nature and difficulty to diagnose, the overall 5-year survival rate of patients with esophageal cancer is $20 \%(4,5)$. Therefore, there is an urgent necessity to discover the molecular mechanisms that lead to the metastasis of esophageal cancer.

MicroRNAs (miRNAs) are a class of small, non-coding, single-stranded RNAs that are ubiquitously expressed in eukaryotic cells (6). Through directly binding to the 3' untranslated region (UTR) of target gene mRNA, miRNAs induce degradation of mRNA or inhibit mRNA translation, resulting in downregulation of target gene expression (7). Previous studies have revealed that mRNA-miRNA regulatory networks are crucial for normal biological processes, including cell differentiation, migration and apoptosis (8-10). Dysregulation of miRNA expression contributes to a number of human diseases such as cancer (11). A microarray study has demonstrated that the expression levels of several miRNAs are promising biomarkers for esophageal cancer (12). miRNA (miR)-542-3p downregulation has been reported in several cancer types $(13,14)$; however, the potential role and molecular mechanism of miR-542-3p in esophageal cancer remains to be determined.

Ovarian tumor domain-containing ubiquitin aldehyde-binding protein 1 (OTUB1) is a hydrolase that is able to specifically remove ubiquitin from proteins to prevent protein degradation (15). By upregulating the expression of oncogenes, OTUB1 facilitates tumor progression in several types of cancers (15-17). In esophageal cancer, OTUB1 stabilizes Snail protein to promote the metastasis of cancer cells (18); however, it is unknown how OTUB1 is regulated in esophageal cancer.

In the present study, miR-542-3p and OTUB1 mRNA expression levels were examined in normal and tumor tissues from patients with esophageal cancer. A negative correlation was observed between miR-542-3p and OTUB1 mRNA expression in tumor tissues. Western blotting and reverse transcription-quantitative PCR (RT-qPCR) data indicated that $O T U B 1$ was negatively regulated by $\mathrm{miR}-542-3 \mathrm{p}$ in esophageal cancer cells. A dual-luciferase assay validated $O T U B 1$ as a target gene for miR-542-3p. Furthermore, function assays demonstrated that miR-542-3p inhibited the migration and invasion of KYSE150 human esophageal squamous cell carcinoma cells through repression of OTUB1 expression. The data from the present study suggested a potential tumor suppressor role for miR-542-3p in esophageal cancer. 


\section{Materials and methods}

Tissue collection. Tumor tissues and matched normal tissues were collected from 40 patients (mean age $56.33 \pm 7.21$, male:female $=25: 15$ ) with esophageal squamous cell carcinoma or esophageal adenocarcinoma in Sheyang People's Hospital (Sheyang, China) between June 2015 and July 2017. Written consent was provided by all participants before the experiments and all procedures were approved by the Ethics Committee of Sheyang People's Hospital (IRB no. SYPH1506). Tissues were stored in $-80^{\circ} \mathrm{C}$ upon collection.

Cell culture. Human esophageal squamous cell carcinoma cell line KYSE150 was purchased from American Type Culture Collection and used within 6 months. Cells were maintained in DMEM (Sigma-Aldrich, Merck KGaA) supplemented with $10 \%$ FBS (Gibco; Thermo Fisher Scientific, Inc.) at $37^{\circ} \mathrm{C}$.

Overexpression and downregulation of miR-542-3p. miR-542-3p mimic (5'-UGUGACAGAUUGAUAACUGAAA-3'), miR-negative control (NC) mimic (5'-AAUUCUCCGAACGUGUCA CTT-3'), miR-542-3p inhibitor (5'-UUUCAGUUAUCAAUC UGUCACA-3') and miR-NC inhibitor (5'-GUGACACGU UCGGAGAAUUTT-3') were synthesized by and purchased from Shanghai GenePharma Co., Ltd. For overexpression or downregulation of miR-542-3p, miR-542-3p mimic (50 nM) or miR-542-3p inhibitor (50 $\mathrm{nM}$ ) was mixed with Lipofectamine ${ }^{\circledR}$ 3000 (Invitrogen; Thermo Fisher Scientific, Inc.) in serum-free DMEM medium for $15 \mathrm{~min}$. The mixtures were added into each well in 6-well plates and incubated for $48 \mathrm{~h}$ before the cells $\left(2 \times 10^{5}\right)$ were harvested for subsequent experiments.

$R N A$ extraction and RT- $q P C R$. Total RNA from cell lines $\left(1 \times 10^{4}\right)$ or tissue samples (50-100 mg) was extracted using TRIzol reagent (Invitrogen; Thermo Fisher Scientific, Inc.) according to the manufacturer's protocol. For mRNA quantification, RNA was reverse-transcribed into first-stranded cDNA using PrimeScript RT Master Mix (Takara Bio, Inc.). RT-qPCR was conducted using a SYBR-Green qPCR Master Mix kit (Takara Bio, Inc.) on an ABI PRISM 7900HT Real-Time PCR System (Applied Biosystems; Thermo Fisher Scientific, Inc.) with the followed thermocycling parameters: Initial denaturation at $95^{\circ} \mathrm{C}$ for $2 \mathrm{~min}$, 40 cycles of $95^{\circ} \mathrm{C}$ for $15 \mathrm{sec}$ and $64^{\circ} \mathrm{C}$ for $30 \mathrm{sec}$. For miRNA quantification, RNA was reverse-transcribed with a Mir-X miRNA First Strand Synthesis kit (Takara Bio, Inc.) followed by qPCR with Mir-X ${ }^{\mathrm{TM}}$ miRNA qRT-PCR SYBR kit (Takara Bio, Inc.). Relative expression levels of miRNA and mRNA were calculated using the $2^{-\Delta \Delta C q}$ method (19). GAPDH and U6 served as internal controls for mRNA and miRNA, respectively. The primer sequences were as listed: miR-542-3p forward, 5'-TGT GACAGATTGATAACT-3' and stem-loop RT primer, 5'-GTC GTATCCAGTGCAGGGTCCGAGGTATTCGCACTGGA TACGACCTGCGGTTTCAGT-3'; OTUB1 forward, 5'-TCG GTCCTATACAAGGAGTATGC-3' and reverse, 5'-GGTCTT GCGGATGTACGAGT-3'; U6 forward, 5'-GTGCTCGCTTCG GCAGCACAT-3' and reverse, 5'-AATATGGAACGCTTC ACGAAT-3', GAPDH forward, 5'-GGAGCGAGATCCCTC CAAAAT-3' and reverse, 5'-GGCTGTTGTCATACTTCTCAT GG-3'. Expression level of miR-542-3p was normalized to U6. Expression level of OTUB1 was normalized to GAPDH.
Protein lysate preparation and western blotting. The antibodies used were as follows: OTUB1 (cat. no. A302-917A; 1:1,000; Bethyl Laboratories, Inc.); GAPDH (cat. no. G8795; 1:5,000; Sigma-Aldrich; Thermo Fisher Scientific, Inc.); Snail (cat. no. sc-393172; 1:1,000; Santa Cruz Biotechnology, Inc., CA, USA); horseradish peroxidase-conjugated secondary antibodies against rabbit (cat. no. 7074, 1:10,000; Cell Signaling Technology, Danvers, MA, USA) and mouse (cat. no. 7076, 1:10,000; Cell Signaling Technology). Protein lysates from cells $\left(1 \times 10^{4}\right)$ were prepared using RIPA lysis buffer (Roche Diagnostics GmbH) followed by determination of protein concentration by the BCA method. Then, protein samples $(20 \mu \mathrm{g})$ were separated on an $10 \%$ SDS-PAGE gel and transferred to a PVDF membrane. The, PVDF membrane was blocked by $5 \%$ bovine serum albumin (BSA, Beijing Solarbio Science \& Technology Co., Ltd.) at room temperature for $2 \mathrm{~h}$. Following incubation with the primary antibody at $4^{\circ} \mathrm{C}$ overnight and a secondary antibody at room temperature for $2 \mathrm{~h}$, the bands were visualized using ECL Western Blot Substrate (Pierce; Thermo Fisher Scientific, Inc.). Densitometric analysis was performed by ImageJ software (version 1.8.0; National Institutes of Health). GAPDH was used to normalize expression data.

Dual-luciferase reporter assay. The position 107-114 of 3'UTR of OTUB1 (5'...UUCACCCCCUUCUUCCUGUCA CA...) mRNA containing the putative target site of miR-542-3p (3'AAAGUCAAUAGUUAGACAGUGU) was determined by TargetScan version 7.1 (20) and amplified from the cDNA of KYSE150 cells and ligated into the pGL3-basic vector (Promega Corporation). pGL3-OTUB1-3'UTR-mutant (Mut, 5 '...UUCACCCCCUUCUUCCUGGAACA...) was created by introducing two site mutations into miR-542-3p potential target sites using QuickChangeSite-Directed Mutagenesis kits (Agilent Technologies, Inc.). pGL3-OTUB1-3'UTR-WT (200 ng) or pGL3-OTUB1-3'UTR-Mut (200 ng) was co-transfected with Renilla plasmid into KYSE150 cells using Lipofectamine ${ }^{\circledR}$ 3000 , followed by transfection of miR-NC mimic $(10 \mathrm{nM})$ or miR-542-3p mimic $(10 \mathrm{nM})$ for $48 \mathrm{~h}$ at $37^{\circ} \mathrm{C}$. The Dual-Luciferase Reporter Assay System (Promega Corporation) was used to measure the relative luciferase activity of each well. The firefly luciferase expression was normalized to Renilla.

OTUB overexpression plasmid construction and transfection. Full-length $O T U B 1$ cDNA was amplified from KYSE150 cells and ligated to a pcDNA3.1 vector (https://www.70dir. com/seo/report_www_youbio_cn.html, YouBio) with the restriction sites of $K p n \mathrm{I}$ and $X h o I$. For overexpression of $O T U B 1$, pcDNA3.1-OTUB1 was mixed with Lipofectamine ${ }^{\circledR} 3000$ (Invitrogen; Thermo Fisher Scientific, Inc.) in serum-free DMEM medium for $15 \mathrm{~min}$. The mixtures were then added into each well in 6-well plates $\left(1 \times 10^{6}\right)$ and incubated for $48 \mathrm{~h}$ at $37^{\circ} \mathrm{C}$ before the cells were harvested for subsequent experiments.

Cell migration assay. A wound healing assay was used to examine the migratory ability of transfected KYSE150 cells. Briefly, $1 \times 10^{6}$ cells were seeded in each well of 6 -well plates. Following transfection, as aforementioned, the cells were cultured in $37^{\circ} \mathrm{C}$ to $90-100 \%$ confluence. A wound area was made in the center of each well using a $10 \mu \mathrm{l}$ pipette tip. The culture medium of the cells was replaced with serum-free 
A
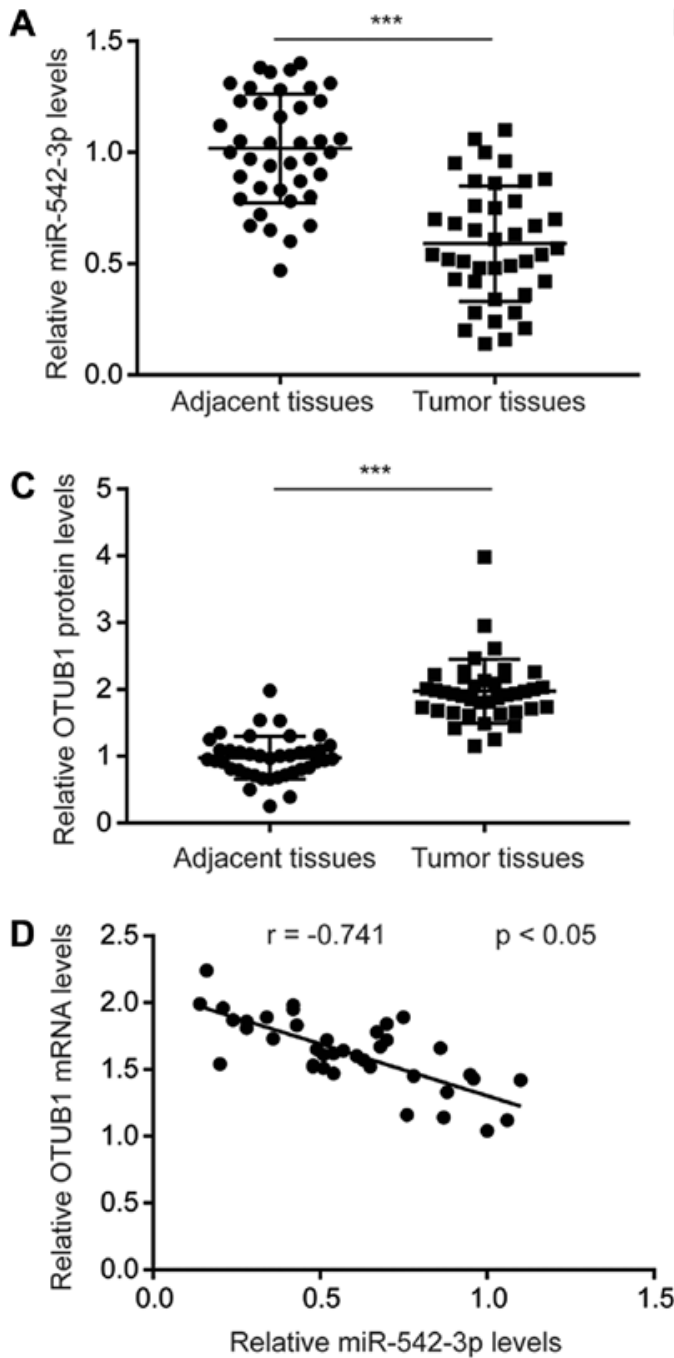
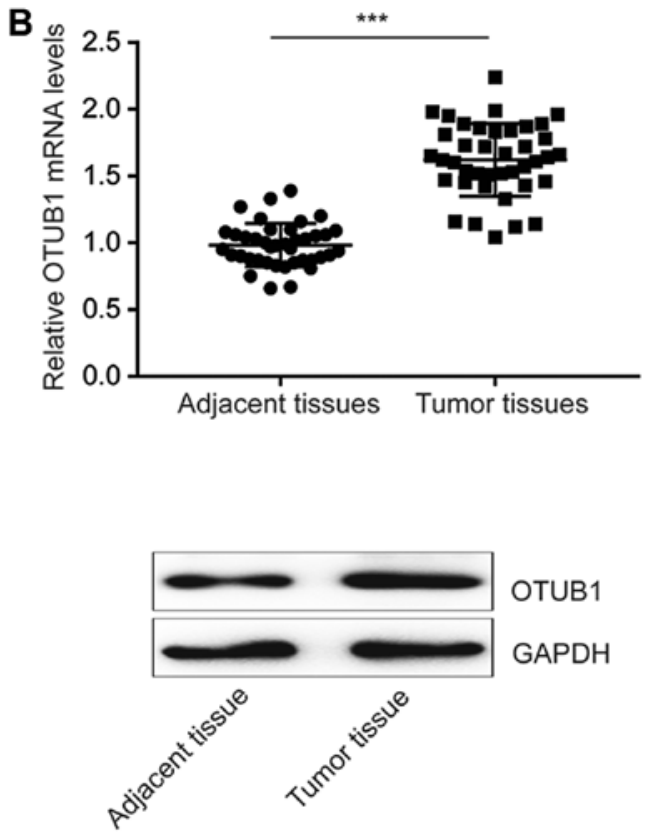

Figure 1. miR-542-3p expression is decreased in esophageal tumor tissues. (A) Compared with adjacent normal tissues, expression of miR-542-3p was decreased in tumor tissues from 40 patients with esophageal cancer. (B and C) Compared with adjacent normal tissues, expression of OTUB1 (B) mRNA and (C) protein was increased in tumoral tissues from 40 patients with esophageal cancer. (D) Expression of miR-542-3p was negatively correlated with OTUB1 mRNA expression in esophageal tumor tissues. ${ }^{* * *} \mathrm{P}<0.0001$. miR-542-3p, microRNA-542-3p; OTUB1, ovarian tumor domain-containing ubiquitin aldehyde-binding protein 1 .

medium. Images were captured using an inverted microscope (Nikon Corporation) at 0 and $24 \mathrm{~h}$ to observe the cells (magnification, x100) that migrated into the wound area. The migrated areas were quantified using Image Pro Plus and normalized to the miR-NC mimic + pcDNA3.1 group.

Cell invasion assay. For the cell invasion assay, BD Matrigel Invasion Chambers (8- $\mu \mathrm{m}$ pore; BD Biosciences) were used. In brief, KYSE150 cells $\left(1 \times 10^{5}\right)$ transfected with miR-NC mimic + pcDNA3.1, miR-542-3p mimic + pcDNA3.1 or miR-542-3p mimic + pcDNA3.1-OTUB1 were cultured in the upper chamber in serum-free DMEM at $37^{\circ} \mathrm{C}$. Following $72 \mathrm{~h}$ of invasion, cells on the upper side of the filter were removed and cells that invaded to the underside of the membranes were fixed using $8 \%$ formaldehyde at room temperature for $15 \mathrm{~min}$, followed by staining with crystal violet at room temperature for $30 \mathrm{~min}$. The number of invaded cells (x400) was counted using a light microscope (Olympus Corporation).

Statistical analysis. Data were analyzed with GraphPad Prism 7 (GraphPad Software, Inc.) and presented as the mean \pm standard error of the mean. For in vitro experiments, the statistical differences were evaluated using Student's t-test (two groups) or using ANOVA followed by Newman Keuls method (three groups). For ex vivo experiments in tumor tissues, the statistical analyses were performed using paired Student's t-test (two groups). Pearson's correlation analysis was performed to determine the correlation between miR-542-3p and $O T U B 1$ mRNA expression levels in esophageal tumor tissues. $\mathrm{P}<0.05$ was considered to indicate a statistically significant difference. All experiments were performed thrice.

\section{Results}

miR-542-3p is downregulated in esophageal tumor tissues. To investigate the role of miR-542-3p in esophageal cancer, RT-qPCR was performed to detect miR-542-3p expression levels in tumoral and adjacent normal tissues from 40 patients with esophageal cancer. The results demonstrated that the expression of miR-542-3p was significantly downregulated in tumor tissues compared with adjacent normal tissues (Fig. 1A). 
A

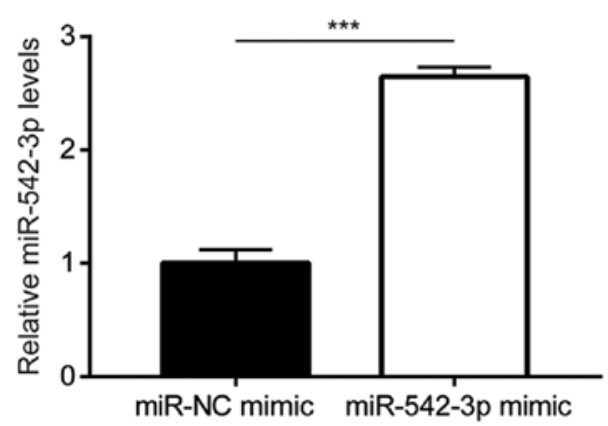

C

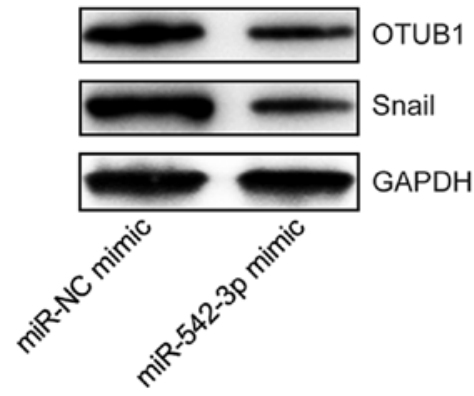

D

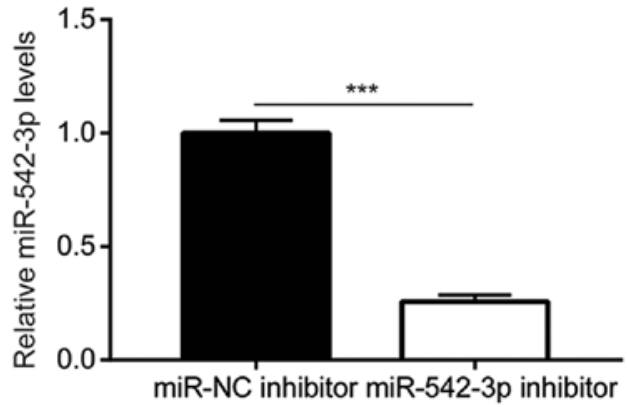

$\mathbf{F}$

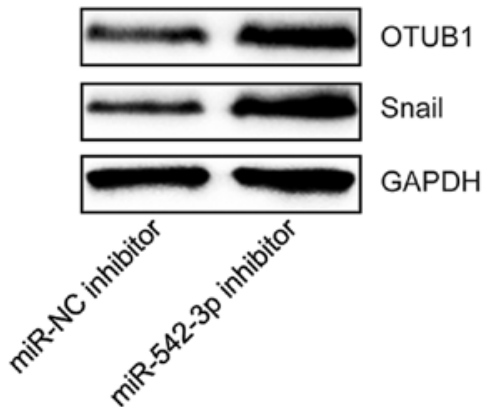

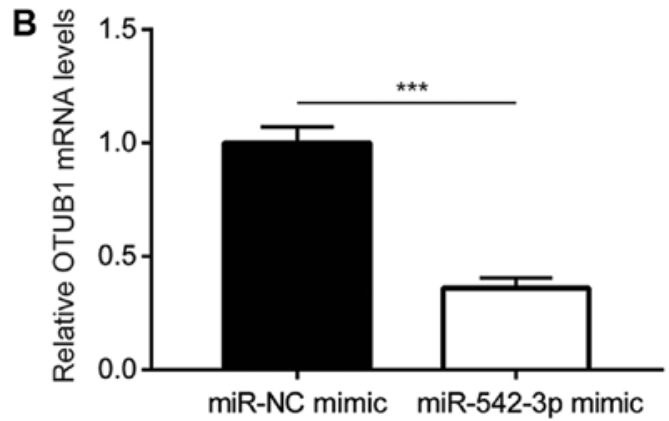

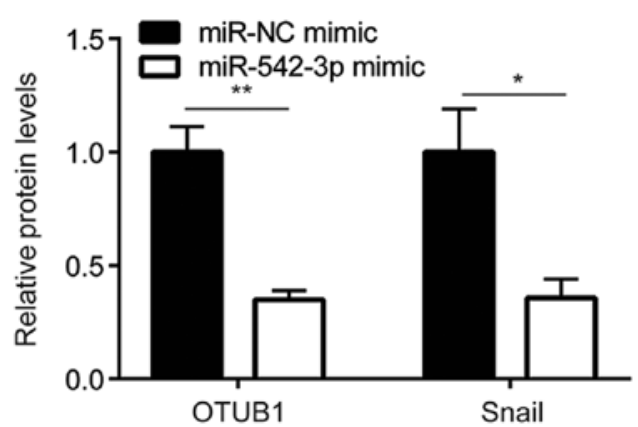

E
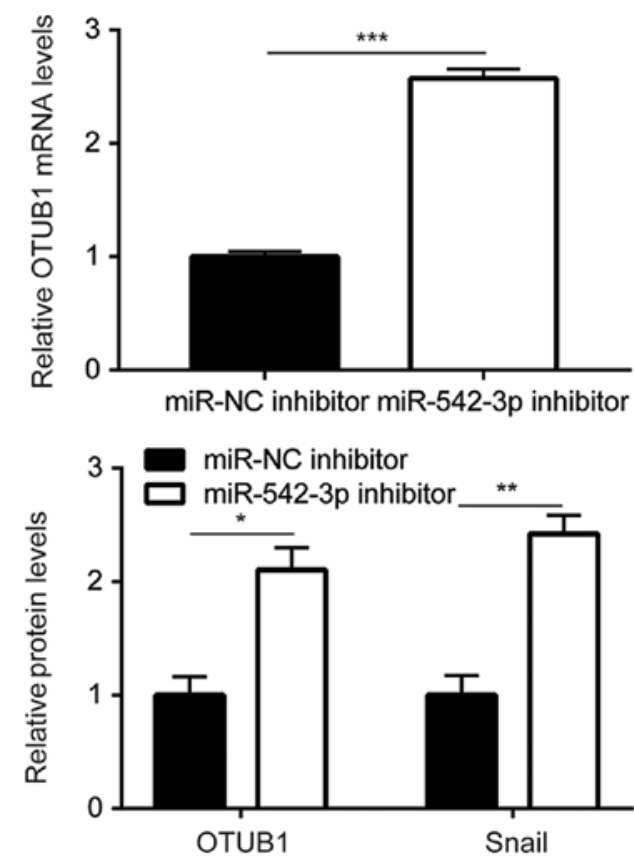

Figure 2. miR-542-3p represses $O T U B 1$ expression in esophageal cancer cells. (A) Transfection with miR-542-3p mimic successfully increased miR-542-3p expression in KYSE150 cells. (B and C) Overexpression of miR-542-3p decreased (B) OTUB1 mRNA and (C) OTUB1 and Snail protein expression in KYSE150 cells. (D) Transfection of miR-542-3p inhibitor successfully decreased miR-542-3p expression in KYSE150 cells. (E and F) Downregulation of miR-542-3p increased (E) OTUB1 mRNA and (F) OTUB1 and Snail protein expression in KYSE150 cells. ${ }^{*} \mathrm{P}<0.05,{ }^{* *} \mathrm{P}<0.01$ and ${ }^{* * *} \mathrm{P}<0.0001$. miR-542-3p, microRNA-542-3p; NC, negative control; OTUB1, ovarian tumor domain-containing ubiquitin aldehyde-binding protein 1.

OTUBI is a newly identified oncogene in esophageal cancer and has been reported to be regulated by miR-542-3p in colorectal cancer $(13,18)$.

In the present study, OTUBI mRNA levels were significantly increased in tumor tissues compared with adjacent normal tissues (Fig. 1B). Western blotting revealed that the protein expression of OTUB1 was also significantly increased in tumor tissues compared adjacent normal tissues (Fig. 1C). In addition, Pearson's correlation analysis indicated that expression of miR-542-3p was significantly negatively correlated with OTUBI mRNA expression levels in esophageal tumor tissues (Fig. 1D).
These results suggested that miR-542-3p may inhibit esophageal cancer progression and act as a tumor suppressor.

Expression of OTUB1 is repressed by miR-542-3p in esophageal cancer cells. To further study the regulatory association between OTUBI and miR-542-3p, miR-542-3p mimic was used to elevate miR-542-3p expression in KYSE150 cells. Compared with cells transfected with miR-NC mimic, transfection with miR-542-3p mimic significantly increased miR-542-3p expression in KYSE150 cells (Fig. 2A). Overexpression of miR-542-3p significantly decreased OTUB1 mRNA levels and 

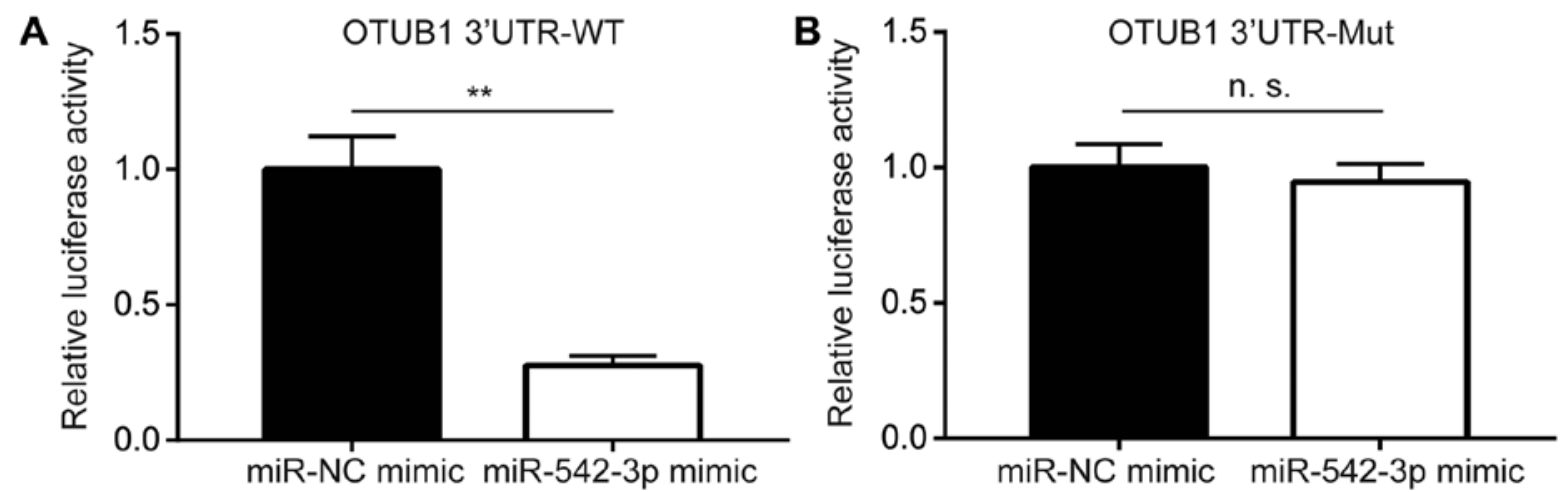

Figure 3. OTUB1 is a target gene for miR-542-3p in esophageal cancer cells. (A) miR-542-3p mimic reduced luciferase activity of KYSE150 cells transfected with OTUB1 3'UTR-WT. (B) miR-542-3p mimic did not alter luciferase activity of KYSE150 cells co-transfected with OTUB1 3'UTR-Mut. "* P $<0.01$. miR-542-3p, microRNA-542-3p; Mut, mutant; NC, negative control; n.s., not significant; OTUB1, ovarian tumor domain-containing ubiquitin aldehyde-binding protein 1; UTR, untranslated region; WT, wild-type.

\section{A}

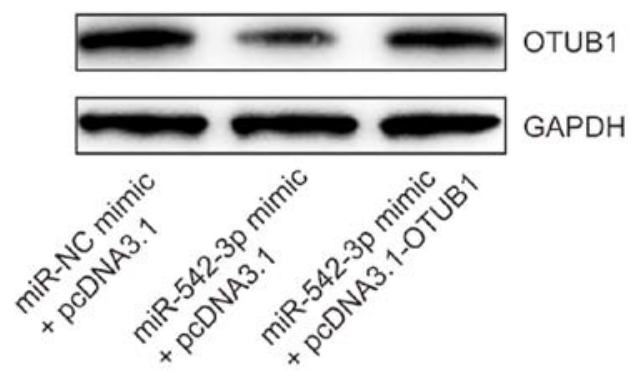

B miR-NC mimic miR-542-3p mimic miR-542-3p mimic + pcDNA3.1 + pcDNA3.1 + pcDNA3.1-OTUB
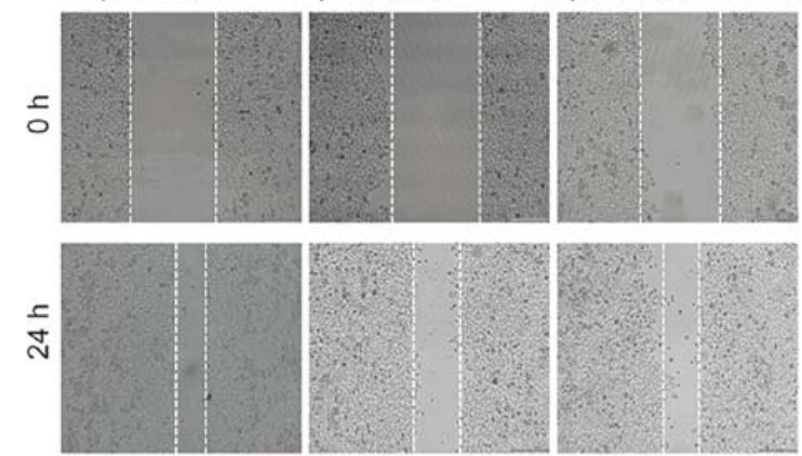
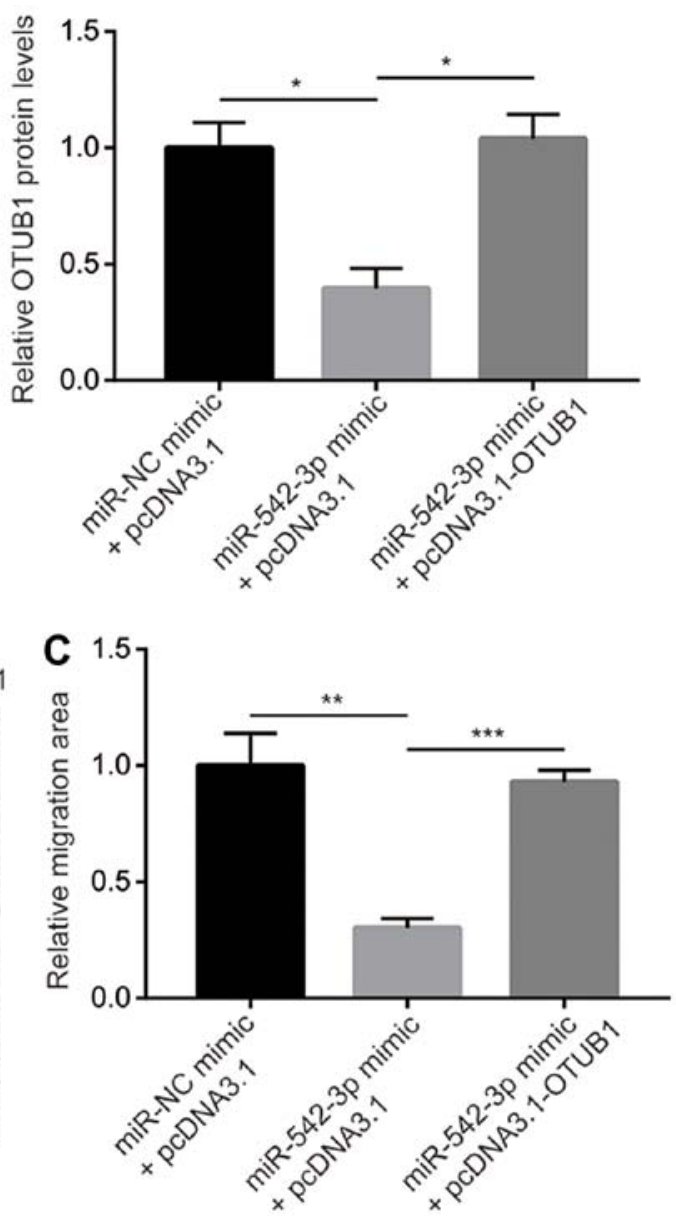

Figure 4. miR-542-3p inhibits migration of KYSE150 cells through the regulation of OTUB1. (A) Overexpression of miR-542-3p reduced OTUB1 protein expression in KYSE150 cells, which was reversed by co-transfection of recombinant OTUB1. (B) Overexpression of miR-542-3p inhibited the migratory ability of KYSE150 cells, which was reversed by co-transfection of recombinant OTUB1. (C) The migrated areas in B were quantified using Image Pro Plus and normalized to the miR-NC mimic + pcDNA3.1 group. ${ }^{*} \mathrm{P}<0.05,{ }^{* *} \mathrm{P}<0.01$ and ${ }^{* * * *} \mathrm{P}<0.0001$. miR-542-3p, microRNA-542-3p; NC, negative control; $O T U B 1$, ovarian tumor domain-containing ubiquitin aldehyde-binding protein 1.

OTUB1 protein levels in cells (Fig. 2B and C). Conversely, transfection with miR-542-3p inhibitor significantly decreased miR-542-3p expression in KYSE150 cells and led to an increased expression of $O T U B 1$ at $\mathrm{mRNA}$ and protein levels (Fig. 2D-F). These data demonstrated that miR-542-3p may negatively regulate $O T U B 1$ in esophageal cancer cells.
OTUB1 is a target gene of miR-542-3p in esophageal cancer cells. To confirm whether miR-542-3p directly regulated OTUB1 expression, dual-luciferase reporter assay was performed in KYSE150 cells. Transfection of miR-542-3p mimic significantly reduced luciferase activity of OTUB1 3'UTR-WT compared with cells transfected with miR-NC 
A

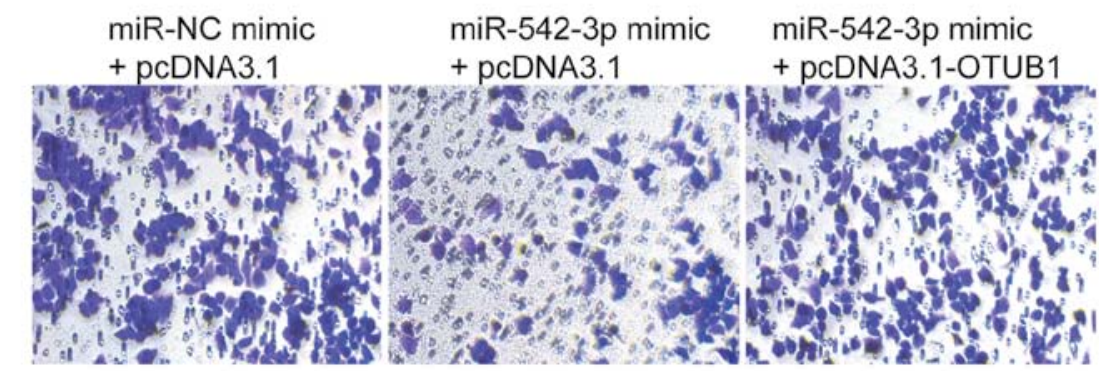

B

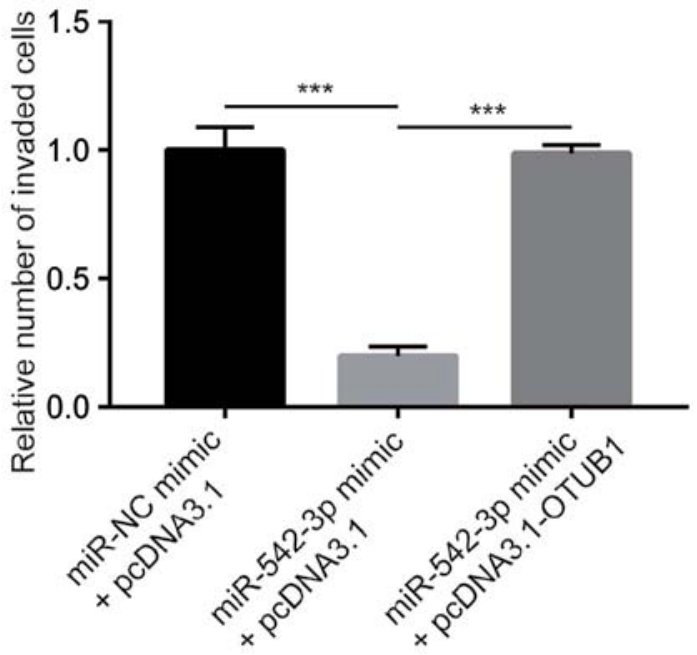

Figure 5. miR-542-3p inhibits invasion of KYSE150 cells. (A) Overexpression of miR-542-3p inhibited the invasive ability of KYSE150 cells, which was reversed by co-transfection with recombinant $O T U B 1$. (B) Quantification of relative invaded cell number in A. ${ }^{* * *} \mathrm{P}<0.0001$. miR-542-3p, microRNA-542-3p; $O T U B 1$, ovarian tumor domain-containing ubiquitin aldehyde-binding protein 1.

mimic (Fig. 3A), whereas overexpression of miR-542-3p did not alter the luciferase activity of OTUB1 3'UTR-Mut (Fig. 3B). These data suggested that miR-542-3p may bind directly to the putative binding site in $3^{\prime} \mathrm{UTR}$ of $O T U B 1$ to repress its expression.

miR-542-3p inhibits esophageal cancer cell migration and invasion through regulation of OTUB1. To study the biological function of miR-542-3p in esophageal cancer cells, wound healing and cell invasion assays were performed to detect cell migratory and invasive ability of cells transfected with the miR-542-3p mimic either with or without overexpression of OTUB1.

Transfection of miR-542-3p mimic reduced OTUB1 protein expression whereas co-transfection of miR-542-3p mimic and recombinant $O T U B 1$ reversed the downregulation of OTUB1 in KYSE150 cells (Fig. 4A). In the wound healing assays, overexpression of miR-542-3p significantly inhibited cell migration towards the wound areas, which indicated that the migration ability of cells was reduced (Fig. 4B and C). In addition, overexpression of OTUB1 reversed migration inhibition induced by miR-542-3p mimic (Fig. 4B and C). Similarly, overexpression of miR-542-3p significantly inhibited the invasive ability of the cells, which was reversed by overexpression of OTUB1 (Fig. 5). The results demonstrated that miR-542-3p may inhibit the migratory and invasive abilities of esophageal cancer cells through repression of $O T U B 1$.

\section{Discussion}

miRNAs regulate a number of oncogenes and tumor suppressors in cells, which can lead to the initiation and progression of cancer $(21,22)$. Several miRNAs have been identified as key regulators of esophageal cancer development and accurate predictors of clinical outcome for patients with esophageal cancer (23-25). Decreased expression of miR-542-3p has been observed in certain types of cancers, including hepatocellular carcinoma, osteosarcoma, colorectal cancer and melanoma $(13,26-29)$. In the present study, miR-542-3p expression and function were investigated in esophageal cancer. RT-qPCR revealed that miR-542-3p expression was decreased in tumor tissues compared with adjacent normal tissues from patients with esophageal cancer. In KYSE150 cells, overexpression of miR-542-3p markedly reduced cell migratory and invasive abilities. Thus, consistent with its tumor suppressor role in other cancer types, miR-542-3p may also function as a tumor suppressor in esophageal cancer.

OTUB1 is a cysteine protease that removes ubiquitin from modified proteins to stabilize target proteins (30). OTUB1 is involved in the regulation of several proteins that are pivotal for the progression of cancer, such as estrogen receptor, p53 and forkhead box M1 $(17,30,31)$. Recently, OTUB1 was demonstrated to promote the metastasis of esophageal cancer by stabilizing Snail (18). miR-542-3p has been demonstrated to bind to 3'UTR of OTUB1 mRNA to downregulate $O T U B 1$ in colorectal cancer cells (13). 
Consistent with the previous study, an increase in $O T U B 1$ mRNA expression was observed in tumor tissues compared with adjacent normal tissues from patients with esophageal cancer. Additionally, OTUB1 expression levels were inversely correlated with miR-542-3p expression levels in tumor tissues, and in KYSE150 cells, overexpression of miR-542-3p decreased OTUB1 expression.

Snail is a member of the Snail superfamily, which functions in cell survival and cell differentiation of cancer cells (32). Furthermore, the development and metastasis of cancer were blocked by Snail suppression (33). Recently, Snail silencing was found to inhibit cell migration of esophageal cells (34). Notably, in KYSE150 cells, overexpression of miR-542-3p decreased Snail expression.

Finally, cell migration and invasion inhibition induced by miR-542-3p overexpression was partially attenuated by co-transfection of recombinant $O T U B 1$ in KYSE150 cells. These results demonstrated that miR-542-3p may regulate $O T U B 1$ to inhibit cell metastasis of esophageal cancer, which were consistent with a previous study about the role of $O T U B 1$ in esophageal cancer (18). Future studies are needed to determine whether the expression of miR-542-3p may be used as a biomarker to predict distant metastasis and overall survival of patients with esophageal cancer.

The results of the present study indicated a potential tumor suppressor role for miR-542-3p in esophageal cancer. Overexpression of miR-542-3p inhibited migration and invasion of esophageal cancer cells. Therefore, upregulation of miR-542-3p may be a potential treatment approach for patients with esophageal cancer.

\section{Acknowledgements}

Not applicable.

\section{Funding}

No funding was received.

\section{Availability of data and materials}

The datasets used and or/analyzed during the current study are available from the corresponding author on reasonable request.

\section{Authors' contributions}

JuS and WY designed the study. JuS, JiS and YD acquired and interpreted the data. YD and JiS collected clinical samples. WY prepared the manuscript and supervised the study.

\section{Ethics approval and consent to participate}

The present study was approved by the Ethics Committee of Sheyang People's Hospital (Sheyang, China). All patients signed written informed consent.

\section{Patient consent for publication}

Not applicable.

\section{Competing interests}

The authors declare that they have no competing interests.

\section{References}

1. Torre LA, Bray F, Siegel RL, Ferlay J, Lortet-Tieulent J and Jemal A: Global cancer statistics, 2012. CA Cancer J Clin 65: 87-108, 2015.

2. Siegel RL, Miller KD and Jemal A: Cancer statistics, 2017. CA Cancer J Clin 67: 7-30, 2017.

3. Rustgi AK and El-Serag HB: Esophageal carcinoma. N Engl J Med 371: 2499-2509, 2014.

4. Siegel R, Naishadham D and Jemal A: Cancer statistics for Hispanics/Latinos, 2012. CA Cancer J Clin 62: 283-298, 2012.

5. Deng HY, Alai G, Luo J, Li G, Zhuo ZG and Lin YD: Cancerous esophageal stenosis before treatment was significantly correlated to poor prognosis of patients with esophageal cancer: A meta-analysis. J Thorac Dis 10: 4212-4219, 2018.

6. Bartel DP: MicroRNAs: Genomics, biogenesis, mechanism, and function. Cell 116: 281-297, 2004.

7. Bartel DP: MicroRNAs: Target recognition and regulatory functions. Cell 136: 215-233, 2009.

8. Chen CZ: MicroRNAs as oncogenes and tumor suppressors. N Engl J Med 353: 1768-1771, 2005.

9. Xu J, Chen Q, Liu P, Jia W, Chen Z and Xu Z: Integration of mRNA and miRNA analysis reveals the molecular mechanism underlying salt and alkali stress Tolerance in tobacco. Int J Mol Sci 20: E2391, 2019.

10. Ye Y, Li SL and Wang SY: Construction and analysis of mRNA, miRNA, IncRNA, and TF regulatory networks reveal the key genes associated with prostate cancer. PLoS One 13: e0198055, 2018.

11. Wang Y, Liang Y and Lu Q: MicroRNA epigenetic alterations: Predicting biomarkers and therapeutic targets in human diseases. Clin Genet 74: 307-315, 2008.

12. Feber A, Xi L, Luketich JD, Pennathur A, Landreneau RJ, Wu M, Swanson SJ, Godfrey TE and Litle VR: MicroRNA expression profiles of esophageal cancer. J Thorac Cardiovasc Surg 135: 255-260; discussion 260, 2008.

13. Yuan L, Yuan P, Yuan H, Wang Z, Run Z, Chen G, Zhao P and $\mathrm{Xu}$ B: miR-542-3p inhibits colorectal cancer cell proliferation, migration and invasion by targeting OTUB1. Am J Cancer Res 7: 159-172, 2017.

14. Althoff K, Lindner S, Odersky A, Mestdagh P, Beckers A, Karczewski S, Molenaar JJ,Bohrer A, Knauer S, Speleman F, et al: miR-542-3p exerts tumor suppressive functions in neuroblastoma by downregulating Survivin. Int J Cancer 136: 1308-1320, 2015.

15. Karunarathna U, Kongsema M, Zona S, Gong C, Cabrera E, Gomes AR, Man EP, Khongkow P, Tsang JW, Khoo US, et al: OTUB1 inhibits the ubiquitination and degradation of FOXM1 in breast cancer and epirubicin resistance. Oncogene 35: 1433-1444, 2016.

16. Baietti MF, Simicek M, Abbasi Asbagh L, Radaelli E, Lievens S, Crowther J, Steklov M, Aushev VN, Martínez García D, Tavernier J and Sablina AA: OTUB1 triggers lung cancer development by inhibiting RAS monoubiquitination. EMBO Mol Med 8: 288-303, 2016

17. Wang Y, Zhou X, Xu M, Weng W, Zhang Q, Yang Y, Wei P and Du X: OTUB1-catalyzed deubiquitination of FOXM1 facilitates tumor progression and predicts a poor prognosis in ovarian cancer. Oncotarget 7: 36681-36697, 2016.

18. Zhou H, Liu Y, Zhu R, Ding F, Cao X, Lin D and Liu Z: OTUB1 promotes esophageal squamous cell carcinoma metastasis through modulating Snail stability. Oncogene 37: 3356-3368, 2018.

19. Livak KJ and Schmittgen TD: Analysis of relative gene expression data using real-time quantitative PCR and the 2(-Delta Delta C(T)) method. Methods 25: 402-408, 2001.

20. Agarwal V, Bell GW, Nam J and Bartel DP: Predicting effective microRNA target sites in mammalian mRNAs. Elife: Aug 4, 2015 (Epub ahead of print). doi: 10.7554/eLife.05005.

21. Lee K and Ferguson LR: MicroRNA biomarkers predicting risk, initiation and progression of colorectal cancer. World J Gastroenterol 22: 7389-7401, 2016.

22. Paliouras AR, Monteverde T and Garofalo M: Oncogene-induced regulation of microRNA expression: Implications for cancer initiation, progression and therapy. Cancer Lett 421: 152-160, 2018. 
23. Fang Y, Fang D and Hu J: MicroRNA and its roles in esophageal cancer. Med Sci Monit 18: RA22-RA30, 2012.

24. Li X, Wainscott $\mathrm{C}$ and $\mathrm{Xi}$ Y: MicroRNA provides insight into understanding esophageal cancer. Thorac Cancer 2: 134-142, 2011.

25. Qi B, Liu SG, Qin XG, Yao WJ, Lu JG, Guo L, Wang TY, Li HC and Zhao BS: Overregulation of microRNA-212 in the poor prognosis of esophageal cancer patients. Genet Mol Res 13: 7800-7807, 2014

26. Wu W, Dang S, Feng Q, Liang J, Wang Y and Fan N: MicroRNA-542-3p inhibits the growth of hepatocellular carcinoma cells by targeting FZD7/Wnt signaling pathway. Biochem Biophys Res Commun 482: 100-105, 2017.

27. Wu Y, You J, Li F, Wang F and Wang Y: MicroRNA-542-3p suppresses tumor cell proliferation via targeting Smad2 inhuman osteosarcoma. Oncol Lett 15: 6895-6902, 2018.

28. Rang Z, Yang G, Wang YW and Cui F: miR-542-3p suppresses invasion and metastasis by targeting the proto-oncogene serine/threonine protein kinase, PIM1, in melanoma. Biochem Biophys Res Commun 474: 315-320, 2016.

29. Wiener R, DiBello AT, Lombardi PM, Guzzo CM, Zhang X, Matunis MJ and Wolberger C: E2 ubiquitin-conjugating enzymes regulate the deubiquitinating activity of OTUB1. Nat Struct Mol Biol 20: 1033-1039, 2013.
30. Sun XX, Challagundla KB and Dai MS: Positive regulation of p53 stability and activity by the deubiquitinating enzyme Otubain 1. EMBO J 31: 576-592, 2012.

31. Stanisić V, Malovannaya A, Qin J,Lonard DM and O'Malley BW: OTU Domain-containing ubiquitin aldehyde-binding protein 1 (OTUB1) deubiquitinates estrogen receptor (ER) alpha and affects ERalpha transcriptional activity. J Biol Chem 284: 16135-16145, 2009

32. Peinado H, Olmeda D and Cano A: Snail, Zeb and bHLH factors in tumour progression: An alliance against the epithelial phenotype? Nat Rev Cancer 7: 415-428, 2007.

33. Mansoori B, Sandoghchian Shotorbani S and Baradaran B: RNA interference and its role in cancer therapy. Adv Pharm Bull 4: 313-321, 2014.

34. Hemmatzadeh M, Mohammadi H, Babaie F, Yousefi M, Ebrazeh M, Mansoori B, Shanehbandi D and Baradaran B: Snail-1 silencing by siRNA inhibits migration of TE-8 esophageal cancer cells through downregulation of metastasis-related genes. Adv Pharm Bull 8: 437-445, 2018.

cc) (i) $\ominus$ This work is licensed under a Creative Commons Attribution-NonCommercial-NoDerivatives 4.0 International (CC BY-NC-ND 4.0) License. 